\title{
Rates and predictors of uptake of mental health support during the COVID-19 pandemic: an analysis of 26,720 adults in the UK in lockdown
}

\author{
Feifei Buํㅁ $\cdot$ Hei Wan Mak ${ }^{1} \cdot$ Daisy Fancourt ${ }^{1}$
}

Received: 11 December 2020 / Accepted: 10 May 2021 / Published online: 18 May 2021

(c) The Author(s) 2021

\begin{abstract}
Purpose The coronavirus disease 2019 (COVID-19) pandemic has put a great strain on people's mental health. A growing number of studies have shown worsening mental health measures globally during the pandemic. However, there is a lack of empirical study on how people support their mental health during the COVID-19 pandemic. This study aimed to examine a number of formal and informal mental health support. Further, it explored factors that might be associated with the use of different types mental health support.

Methods Data from 26,720 adults in the UCL COVID-19 Social Study were analysed between 13th April 2020 and 3rd July 2020. Data were analysed using logistic and Poisson regression models.

Results About $45 \%$ of people reported talking to friends or family members to support their mental health, $43 \%$ engaging in self-care activities, $20 \%$ taking medication, $9 \%$ speaking to mental health professionals, $8 \%$ talking to a GP or other health professional, and another $8 \%$ using helpline or online services. Gender, education, living status, loneliness, pre-existing mental health conditions, general depression and anxiety, coping and personality were found to be associated with the use of mental health support.

Conclusion While the negative impacts caused by the COVID-19 pandemic are inevitable, people can play an active role in managing their mental health. Understanding the patterns and predictors of various kinds of mental health support during the pandemic is crucial for future service planning and delivery through recognising potential barriers to mental health care faced by certain groups.
\end{abstract}

Keywords Mental health $\cdot$ Support $\cdot$ Service $\cdot$ Coping $\cdot$ COVID-19

\section{Introduction}

The stress and anxiety caused by the emergence of the COVID19 pandemic, the restrictions on social distancing (e.g. quarantine, national lockdown), reduced access to local and health services, changes in working environment and employment schemes (e.g. the furlough scheme), and the closure of leisure events and infrastructures have caused unprecedented impacts on people's mental health and well-being. Multiple studies have highlighted adverse effects on loneliness, stress, anxiety,

Daisy Fancourt

d.fancourt@ucl.ac.uk

1 Department of Behavioral Science and Health, University College London, 1-19 Torrington Place, London WC1E 7HB, UK depression, irritability, confusion, fear, insomnia, guilty and social anxiety [1-4]. The proportion of people with a clinically significant level of mental distress increased by around $43 \%$, from 19 to $27 \%$ during the first UK lockdown [2], with young adults, women, people of lower socio-economic status (SES) and those living with children most negatively affected $[2,5,6]$. Indeed, given the immeasurable cost of personal, social, economic, and health burden during COVID-19, it is expected that many countries (including the UK) will face a mental health pandemic that could last for another few years (as has also been shown in other national crisis such as the Great Recession in the US from 2007 to 2009 [7] and other pandemics [4]). As a consequence, protecting mental health has been recognised as high priority to help individuals build their resilience, adapt to inevitable changes caused by the pandemic, cope with adversity, and to prevent the worsening of 
mental ill-health and the experience of suicidal thoughts, selfharm or suicides [8].

To promote population mental health, health services across the UK have been providing guidelines and information to help people to get access to social care and support during the pandemic. These include helplines, recommended home workout and relaxation techniques [9]. Further, mental health charities, organisations and support groups have been offering formal advice and helpline services to support mental health during the pandemic (e.g. Mental Health Foundation, Samaritans, and YoungMinds). Indeed, a report from Samaritans shows that there was a notable increase in the number of contacts from people who were concerned about COVID-19 and other issues such as finances, social well-being and mental health during lockdown in March 2020 [10]. Additionally, there have been reports of many individuals drawing on informal support, including using mental health apps, engaging in self-care behaviours, and speaking with friends or family about their mental health. However, a crucial question is whether these services have been reaching those most in need. For instance, it has been shown that individuals from ethnic minority and lower socio-economic backgrounds experience more barriers (e.g. financial expense, lack of awareness about how to get help, language barriers) to mental health care outside of pandemic situations [11, 12]. But preliminary research during the pandemic has already suggested that some further high-risk groups are experiencing barriers. For instance, a recent study found that around two in five people engaging in self-harm behaviours and three in five people with self-harm/suicidal thoughts or reporting abuse had not been able to access any type of formal support during lockdown, while nearly half of people who reported abuse, self-harm/suicidal thoughts and self-harm behaviours additionally did not receive any informal support [13]. Therefore, it is vital to ascertain in more detail the demographics of people who have not been accessing either formal or informal support to inform the targeting of further more specific support towards groups who may be facing more barriers to mental health care.

In light of this, this study used a large sample of adults in the UK to examine how engagement with both formal mental health support (e.g. taking medication, speaking to mental health or other health professionals) and informal mental health support (e.g. helpline or online service, self-care or speaking to family/friends) during COVID-19 varied depending on people's demographic backgrounds, socio-economic characteristics, social factors, mental health, coping strategies, and personality.

\section{Methods}

\section{Participants}

Data were drawn from the UCL COVID-19 Social Study; a large panel study of the psychological and social experiences of over 70,000 adults (aged 18+) in the UK during the COVID-19 pandemic. The study commenced on 21st March 2020 involving weekly and then st monthly online data collection from participants for the duration of the COVID-19 pandemic in the UK. While not random, the study has a well-stratified sample that was recruited using three primary approaches. First, convenience sampling was used, including promoting the study through existing networks and mailing lists (including large databases of adults who had previously consented to be involved in health research across the UK), print and digital media coverage, and social media. Second, more targeted recruitment was undertaken through partnership with recruitment companies focussing on (i) individuals from a low-income background, (ii) individuals with no or few educational qualifications, and (iii) individuals who were unemployed. Third, the study was promoted via partnerships with third sector organisations to vulnerable groups, including adults with pre-existing mental illness, older adults, and carers. The study was approved by the UCL Research Ethics Committee [12467/005] and all participants gave informed consent.

In this study, we used data between 13th April 2020 (when mental health support information started being collected) and 3rd July 2020 (by which point lockdown measures in the UK had been substantially eased but leisure and cultural facilities and community centres remained closed). A total number of 58,260 adults participated at least once during this period. Except for demographic variables collected when participants first joined the study, other information were mostly collected weekly. However, the coping variables were measured in a one-off module collected between 7 and 14th May 2020; this was the main reason for data reduction. After restricting the sample to only participants who provided full information on all variables of interests, we had an analytical sample of 26,720 participants.

\section{Measures}

This study looked at a range of strategies that people used to support their mental health. Participants were asked: 'in the past week have you done any of the following to support your mental health?' These included: (1) taking medication (e.g. anti-depressants), (2) speaking to a mental 
health professional (e.g. psychiatrist or psychologist), (3) talking to a GP or other health professional, (4) speaking to someone on a support helpline (e.g. Samaritans or NHS Volunteers) or accessing online mental health programmes or forums (cognitive behavioural therapy), (5) spending time on self-care activities specifically to help their mental health (e.g. mindfulness, meditation or planning time for hobbies or relaxation) or using other self-help resource (e.g. self-help books, videos, apps), and (6) speaking to a family member or friend about their mental health. All were coded a binary variable (yes or no) indicating if participants had used any of these strategies at any point between 13th April and 3rd July 2020. Further, we derived a count variable of the total number of different types of mental health support used by each participant.

To understand how different types of mental health support varied across personal characteristics and background, we considered a wide range of potential predictors. These included demographic and socio-economic factors such as age $(18-29,30-45,46-59,60+)$, gender (women vs. men), ethnicity (Black, Asian, and minority ethnic (ethnic minority) vs. white), education (GCSE or below, A levels or equivalent, degree or above), employment status (employed vs. not employed), annual household income ( $<£ 30,000$ vs. $>£ 30,000)$ and area of living (rural vs. urban). We also considered social factors, which included living status (living alone, living with others including children, living with others, no child), social network (close friends $<3$ vs. $\geq 3$ ) and baseline loneliness level measured by the 3-item UCLA loneliness scale (a short form of the Revised UCLA Loneliness Scale, UCLAR [14]). Our analysis also adjusted for a set of baseline mental health measures indicating whether participants had any pre-existing diagnosed mental health conditions. Also included were depression, which was measured by the Patient Health Questionnaire (PHQ-9; a standard instrument for diagnosing depression in primary care [15]), and anxiety measured by the Generalised Anxiety Disorder (GAD-7; a well-validated tool used to screen and diagnose generalised anxiety disorder in clinical practice and research [16]). Both measured at the first week during the observational period. Moreover, we considered psychological factors including personality traits and coping styles. Personality was measured using the short Big Five Inventory (BFI-2) which comprised subscales on extraversion, neuroticism, openness, conscientiousness and agreeableness [17]. Factor scores for each subscale were derived from confirmatory factor analysis. Coping was measured by the brief COPE Inventory which contains 28 items measuring 14 different types of coping tactics [18]. In this study, we derived coping scores based on the four-factor model, including problem-focussed (e.g. active coping), emotion-focussed (e.g. religion), avoidant (e.g. substance use) and socially supported (e.g. instrumental support) coping strategies [19].

\section{Analysis}

We used logistic regression to estimate the associations between predictors and the use of each type of mental health support. Further, we fitted a Poisson regression model to examine predictors that were associated with the number of strategies that people used to support their mental health. The assumption of equidispersion was checked and additionally tested by comparing our model to negative binomial regression. All data were weighted to the proportions of gender, age, ethnicity, education, and country of living obtained from the Office for National Statistics [20].

In addition to the main analyses, sensitivity analyses were performed using an alternative measure of pre-existing mental health conditions which made use of more specific conditions (e.g. schizophrenia, bipolar disorder, obsessive-compulsive disorder, psychosis, etc.) and were available for a reduced number of participants (80\%). This yielded largely similar findings (see the Supplement). Sensitivity analyses stratified by pre-existing mental health conditions were also provided in the Supplement. Confirmatory factor analyses were fitted in Mplus V8, but the main analyses were conducted using Stata V15.

\section{Results}

\section{Descriptive statistics}

In our weighted sample, $51 \%$ were women and $13 \%$ were from ethnic minority backgrounds. On average, $34 \%$ of the sample had a degree or other higher education qualification, $57 \%$ were in employment, $58 \%$ with a household income under $£ 30,000$, and $21 \%$ lived in a rural area. About one in five participants had a pre-existing mental health diagnosis (see Table S1 in the Supplement).

The most commonly used mental health support during lockdown was talking to friends or family members (45\%), followed by engaging in self-care activities (43\%) and taking medication (20\%). Less common strategies included speaking to mental health professionals (9\%), talking to a GP or other health professional (8\%), and speaking to someone on a support helpline or using an online programme or forum (8\%) (Fig. 1a). The percentage of participants using each type of support was much higher among participants with a pre-existing mental health diagnosis than those without. However, the patterns of more or less commonly used strategies were similar between these two groups. On average, $37 \%$ of participants did not take any action to support their mental health, compared to $24 \%$ of people using one 
Fig. 1 Prevalence of using different types and total number of mental health support by preexisting mental health diagnosis

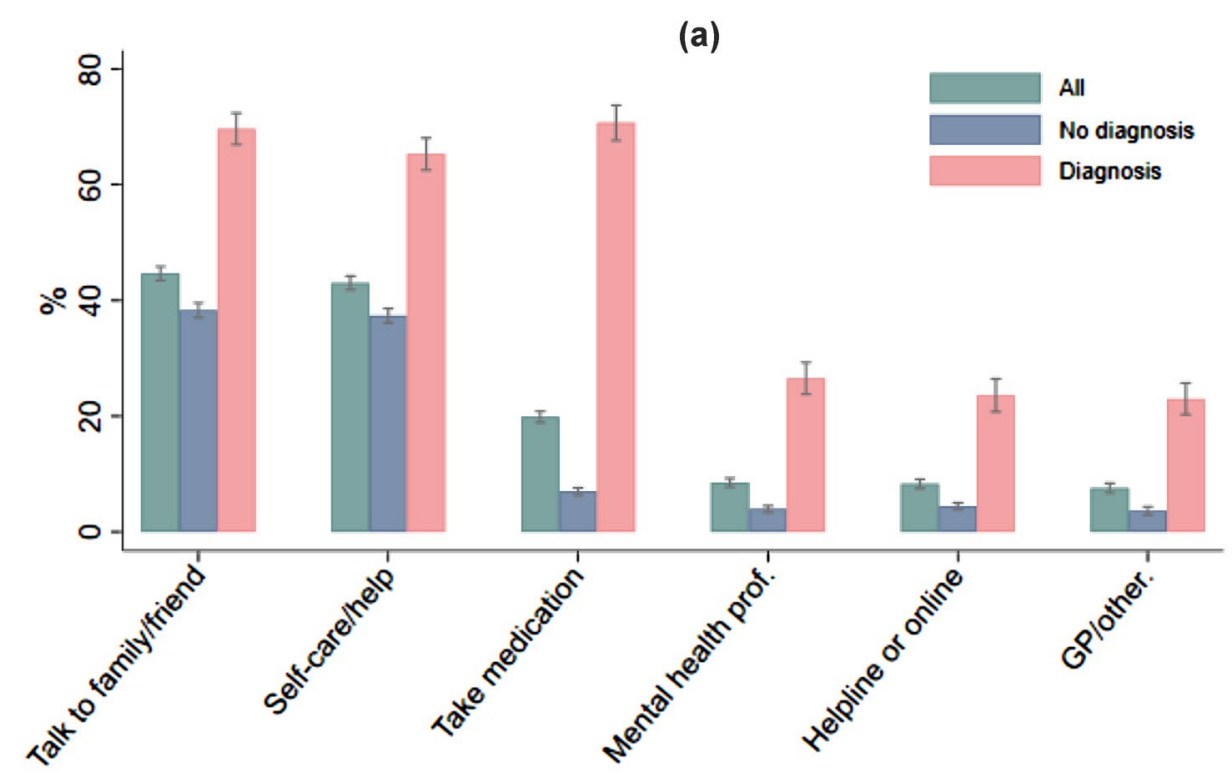

(b)

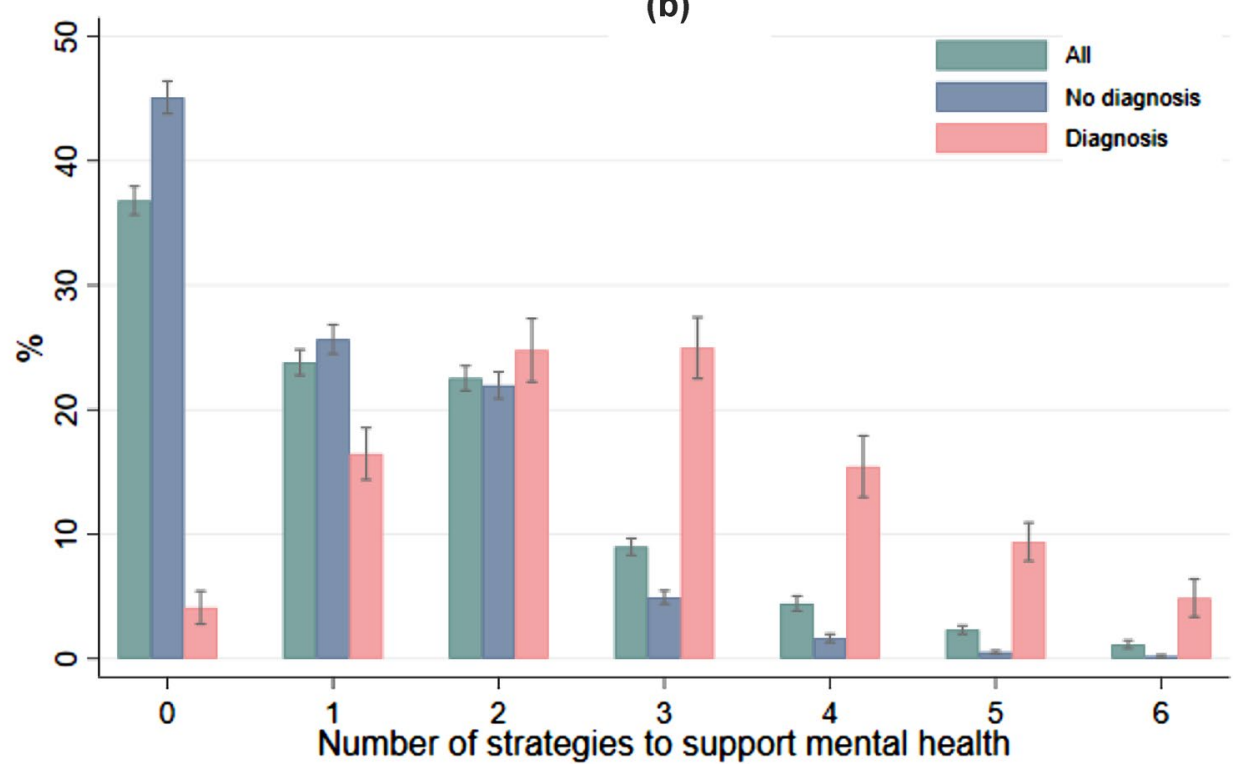

strategy, 23\% using two strategies, and $17 \%$ using three or more strategies to support their mental health (Fig. 1b). Participants with and without a pre-existing mental health diagnosis showed different distribution of total number of strategies. Not surprisingly, the number of different types of support used during the pandemic was higher amongst participants with a mental health condition than those without a condition.

\section{Demographic and socio-economic characteristics}

Comparing to younger adults (aged 18-29), older adults were 2-3 times more likely to take medication to support their mental health (OR: 1.92-2.24). However, they were less likely to speak to mental health professionals, seek help through helplines or online services, engage in selfcare activities or speak with family/friends. Women had a $101 \%$ higher odds of engaging in self-care activities (OR: $2.01,95 \%$ CI: $1.77-2.27$ ) and a $35 \%$ higher odds of speaking to family/friends to support their mental health $(\mathrm{OR}=1.35$, 95\% CI: 1.19-1.53). No gender differences were found in terms of using other mental health support. People from ethnic minority backgrounds were less likely to take medication (OR: 0.64, 95\% CI: 0.42-0.98) but more likely to seek help through helplines or online services (OR: 1.71, 95\% CI: 1.17-2.48). Ethnicity did not predict use of other types of mental health support. People with higher educational levels were less likely to take medication (OR: 0.76-0.78), 
but they were more likely to support their mental health through speaking to mental health professionals (OR: 1.42, 95\% CI: 1.04-1.94), helplines or online services (OR: 1.38-1.41), self-care activities (OR: 1.30-2.01) and talking to family/friends (OR: 1.25-1.42). People with lower household income had a $25 \%$ higher odds of taking medication to support their mental health (OR: 1.25, 95\% CI: 1.04-1.50). Those who were employed were more likely to talk to their family/friends to support good mental health (OR: 1.19, 95\%CI: 1.02-1.38). No association was found between area of living and use of any mental health support (Table 1).

\section{Social factors}

With respect to social factors, people who lived alone had higher odds of talking to mental health professionals (OR: 1.49-1.67), engaging in self-care activities (OR: 1.49, 95\% CI: 1.25-1.82) and talking to family/friends (OR: 1.37-1.43) than those who lived with others. There was no evidence that the size of social network was related to use of mental health support. However, the results show that those who were lonelier were more likely to use most types of mental health support (OR: 1.09-1.18), except for taking medication and talking to family/friends (Table 1).

\section{Mental health}

In relation to mental health, people with pre-existing mental health diagnoses were more likely to use all approaches to support their mental health. In particular, the odds of taking medication for someone with a mental health diagnosis were nearly 25 times of that for those without a diagnosis (OR: 24.53, 95\% CI: 20.08-29.97). Further, people with a higher level of depression were more likely to take medication (OR: 1.06, 95\% CI: 1.04-1.09) and speak to mental health professionals (OR: 1.04, 95\% CI: 1.01-1.07), whereas those with a higher level of anxiety were more likely to speak to a GP/other health professionals (OR: 1.04, 95\% CI: 1.02-1.06) and engage in self-care activities (OR: 1.03, 95\% CI: 1.01-1.05). Higher levels of depression and anxiety were both associated with higher odds of using helpline/ online services and talking to family/friends (Table 1).

\section{Psychological factors}

Regarding psychological factors, people with a problemfocussed coping strategy were more likely to engage in self-care activity (OR: 1.80, 95\% CI: 1.53-2.11), whereas those with an avoidant coping strategy were more likely to take medication (OR: 1.43, 95\% CI: 1.21-1.70). Individuals with an emotion-focussed coping strategy were more likely to support their mental health through medication (OR: 1.17, 95\% CI: $1.00-1.37)$ and self-care (OR: $1.47,95 \%$ CI:
1.32-1.64). A socially supported coping strategy was positively associated with all types of mental health support, in particular talking to family/friends (OR: $4.19,95 \% \mathrm{CI}$ : 3.73-4.72). There was also evidence that personality was associated with using different mental health support. People with higher levels of agreeableness and neuroticism were more likely to seek mental health support through helpline/ online services (OR: 1.27-1.41) and family/friends (OR: 1.22-1.30). People who were more open to experiences or extraverted were also more likely to seek support from family/friends (OR: 1.13-1.35). However, they differed in that openness to experiences was associated with higher odds of self-caring (OR: 1.77, 95\% CI: 1.57-2.01), whereas extraversion with lower odds (OR: 0.88, 95\% CI: 0.80-0.97). Further, while people with higher levels of conscientiousness were less likely to engage in self-care activities, those who were more agreeable or neurotic were likely to seek help through helpline/online services or communicating with family/friends (Table 1).

\section{Number of types of mental health support}

When looking at the number of different mental health approaches people used during COVID-19, results show that people aged 60 or above took fewer approaches to support their mental health compared with younger adults (IRR: 0.66, 95\% CI: 0.62-0.71). In contrast, women (IRR: 1.14, 95\% CI: 1.09-1.20), people with higher educational levels (IRR: 1.08-1.20), those who lived alone (IRR: 1.09-1.13), those with a higher level of loneliness (IRR: $1.05,95 \% \mathrm{CI}$ : 1.03-1.06), depression (IRR: 1.01, 95\% CI: 1.01-1.02) and anxiety (IRR: $1.01,95 \%$ CI: $1.00-1.01$ ), and people with a pre-existing mental health diagnosis (IRR: $1.88,95 \% \mathrm{CI}$ : 1.80-1.97) used more approaches to support their mental health. In addition, people with various coping strategies (IRR: 1.04-1.42) and those with higher levels of openness (IRR: 1.07, 95\% CI: 1.03-1.11), extraversion (IRR: 1.05, 95\% CI: 1.10-1.08), agreeableness (IRR: $1.09,95 \% \mathrm{CI}$ : 1.02-1.17) and neuroticism (IRR: 1.09, 95\% CI: 1.05-1.13) also used various types of mental health support. Finally, ethnicity, employment status, household income, living area, social network and level of conscientiousness were not related to the number of types of mental health support people accessed (Fig. 2).

\section{Discussion}

This study examined what kinds of mental health support individuals accessed during the first UK lockdown due to the COVID-19 pandemic and how this accessing of support was patterned amongst different groups. Informal mental health support such as talking to family/friends and 
Table 1 Results from logit model on each strategy to support mental health including all predictors $(N=26,720)$

\begin{tabular}{|c|c|c|c|c|c|c|}
\hline & \multicolumn{2}{|c|}{$\begin{array}{l}\text { Model I: } \\
\text { Medication }\end{array}$} & \multicolumn{2}{|c|}{$\begin{array}{l}\text { Model II: Mental health profes- } \\
\text { sionals }\end{array}$} & \multicolumn{2}{|c|}{$\begin{array}{l}\text { Model III: } \\
\text { GP/other health profes- } \\
\text { sionals }\end{array}$} \\
\hline & OR & $95 \% \mathrm{CI}$ & OR & $95 \% \mathrm{CI}$ & OR & $95 \% \mathrm{CI}$ \\
\hline 30-45 (vs. 18-29) & 1.92 & [1.38-2.67] & 0.76 & {$[0.57-1.01]$} & 1.04 & {$[0.72-1.50]$} \\
\hline 46-59 (vs. 18-29) & 2.82 & [2.01-3.97] & 0.64 & {$[0.47-0.87]$} & 1.07 & {$[0.72-1.61]$} \\
\hline $60+($ vs. $18-29)$ & 2.24 & {$[1.55-3.25]$} & 0.32 & {$[0.21-0.49]$} & 0.67 & [0.41-1.09] \\
\hline Women (vs. men) & 1.07 & {$[0.86-1.32]$} & 0.93 & {$[0.70-1.24]$} & 0.84 & {$[0.63-1.13]$} \\
\hline Ethnic minority (vs. white) & 0.64 & {$[0.42-0.98]$} & 1.15 & {$[0.75-1.75]$} & 1.08 & {$[0.61-1.90]$} \\
\hline Education medium (vs. low) & 0.76 & {$[0.60-0.96]$} & 1.05 & {$[0.74-1.49]$} & 1.12 & {$[0.82-1.53]$} \\
\hline Education high (vs. low) & 0.78 & {$[0.63-0.97]$} & 1.42 & {$[1.04-1.94]$} & 0.95 & {$[0.72-1.27]$} \\
\hline Employed (vs. not employed) & 0.94 & {$[0.77-1.13]$} & 0.92 & {$[0.72-1.17]$} & 0.81 & {$[0.59-1.11]$} \\
\hline Income $<30 \mathrm{k}$ (vs. $\geq 30 \mathrm{k})$ & 1.25 & {$[1.04-1.50]$} & 0.85 & [0.67-1.09] & 1.08 & {$[0.76-1.53]$} \\
\hline Rural (vs. urban) & 1.11 & {$[0.91-1.35]$} & 1.22 & {$[0.93-1.60]$} & 1.03 & {$[0.79-1.34]$} \\
\hline Living with children (vs. alone) & 1.05 & {$[0.81-1.36]$} & 0.60 & {$[0.44-0.82]$} & 0.89 & {$[0.63-1.26]$} \\
\hline Living with others, no child (vs. alone) & 1.00 & {$[0.81-1.23]$} & 0.65 & {$[0.50-0.85]$} & 0.83 & {$[0.63-1.08]$} \\
\hline Close friends $<3$ (vs. $\geq 3$ ) & 1.10 & {$[0.90-1.35]$} & 1.25 & {$[0.94-1.67]$} & 1.10 & {$[0.78-1.55]$} \\
\hline Loneliness & 1.06 & {$[1.00-1.12]$} & 1.18 & {$[1.10-1.26]$} & 1.13 & {$[1.06-1.20]$} \\
\hline Mental health diagnosis (vs. none) & 24.53 & {$[20.08-29.97]$} & 3.89 & {$[3.02-4.99]$} & 3.13 & {$[2.37-4.14]$} \\
\hline Depression (PHQ-9) & 1.06 & {$[1.04-1.09]$} & 1.04 & {$[1.01-1.07]$} & 1.03 & {$[1.00-1.06]$} \\
\hline Anxiety (GAD-7) & 0.98 & {$[0.95-1.00]$} & 1.02 & {$[0.98-1.05]$} & 1.06 & {$[1.04-1.10]$} \\
\hline Coping: problem-focussed & 0.80 & {$[0.64-1.01]$} & 0.85 & {$[0.65-1.10]$} & 1.10 & {$[0.86-1.41]$} \\
\hline Coping: emotion-focussed & 1.17 & {$[1.00-1.37]$} & 1.08 & {$[0.88-1.32]$} & 0.92 & {$[0.76-1.11]$} \\
\hline Coping: avoidant & 1.43 & {$[1.21-1.70]$} & 1.11 & {$[0.88-1.40]$} & 1.23 & {$[0.96-1.59]$} \\
\hline Coping: socially supported & 1.31 & {$[1.13-1.52]$} & 2.46 & {$[2.06-2.93]$} & 1.67 & {$[1.36-2.05]$} \\
\hline Personality: openness to experience & 0.86 & {$[0.73-1.01]$} & 1.07 & {$[0.87-1.32]$} & 0.92 & {$[0.74-1.13]$} \\
\hline Personality: conscientiousness & 1.07 & {$[0.91-1.25]$} & 0.92 & {$[0.77-1.11]$} & 1.11 & {$[0.88-1.41]$} \\
\hline Personality: extraversion & 1.04 & [0.90-1.19] & 1.14 & {$[0.94-1.39]$} & 1.21 & [0.98-1.49] \\
\hline Personality: agreeableness & 1.32 & {$[0.97-1.80]$} & 1.05 & {$[0.73-1.52]$} & 0.82 & {$[0.55-1.22]$} \\
\hline \multirow[t]{3}{*}{ Personality: neuroticism } & 1.12 & {$[0.97-1.30]$} & 1.24 & {$[0.98-1.57]$} & 1.11 & [0.89-1.37] \\
\hline & \multicolumn{2}{|c|}{ Model IV: Helpline/online services } & \multicolumn{2}{|c|}{$\begin{array}{l}\text { Model V: } \\
\text { Self-care/help }\end{array}$} & \multicolumn{2}{|c|}{ Model VI: family/friends } \\
\hline & OR & $95 \% \mathrm{CI}$ & OR & $95 \% \mathrm{CI}$ & OR & $95 \% \mathrm{CI}$ \\
\hline 30-45 (vs. 18-29) & 1.15 & {$[0.84-1.57]$} & 0.82 & {$[0.66-1.02]$} & 0.80 & {$[0.63-1.02]$} \\
\hline 46-59 (vs. 18-29) & 1.05 & {$[0.79-1.39]$} & 0.63 & {$[0.50-0.78]$} & 0.58 & {$[0.46-0.74]$} \\
\hline $60+($ vs. 18-29) & 0.71 & {$[0.51-0.99]$} & 0.34 & {$[0.27-0.43]$} & 0.34 & {$[0.26-0.43]$} \\
\hline Women (vs. men) & 0.84 & [0.66-1.07] & 2.01 & {$[1.77-2.27]$} & 1.35 & {$[1.19-1.53]$} \\
\hline Ethnic minority (vs. white) & 1.71 & {$[1.18-2.48]$} & 1.01 & {$[0.78-1.31]$} & 0.97 & {$[0.75-1.24]$} \\
\hline Education medium (vs. low) & 1.41 & {$[1.03-1.94]$} & 1.30 & {$[1.09-1.55]$} & 1.25 & {$[1.04-1.50]$} \\
\hline Education high (vs. low) & 1.38 & {$[1.00-1.92]$} & 2.01 & {$[1.68-2.41]$} & 1.42 & {$[1.19-1.71]$} \\
\hline Employed (vs. Not employed) & 0.99 & {$[0.79-1.24]$} & 1.08 & {$[0.94-1.25]$} & 1.19 & {$[1.02-1.38]$} \\
\hline Income < $30 \mathrm{k}$ (vs. $\geq 30 \mathrm{k})$ & 1.08 & {$[0.86-1.37]$} & 0.97 & {$[0.84-1.11]$} & 0.94 & {$[0.82-1.09]$} \\
\hline Rural (vs. urban) & 1.03 & {$[0.82-1.28]$} & 1.00 & {$[0.89-1.13]$} & 0.95 & {$[0.84-1.09]$} \\
\hline Living with children (vs. alone) & 1.01 & {$[0.74-1.37]$} & 0.67 & {$[0.55-0.80]$} & 0.70 & {$[0.58-0.85]$} \\
\hline Living with others, no child (vs. alone) & 0.96 & {$[0.73-1.26]$} & 0.90 & {$[0.78-1.04]$} & 0.73 & {$[0.63-0.85]$} \\
\hline Close friends $<3$ (vs. $\geq 3$ ) & 1.02 & {$[0.77-1.36]$} & 0.96 & {$[0.84-1.11]$} & 0.87 & {$[0.75-1.01]$} \\
\hline Loneliness (UCLA-3) & 1.17 & {$[1.09-1.25]$} & 1.09 & {$[1.05-1.13]$} & 1.03 & {$[0.98-1.07]$} \\
\hline Mental health diagnosis (vs. none) & 2.76 & {$[2.16-3.52]$} & 2.05 & {$[1.73-2.43]$} & 2.06 & [1.74-2.45] \\
\hline Depression (PHQ-9) & 1.03 & [1.00-1.06] & 0.99 & [0.98-1.01] & 1.04 & {$[1.02-1.06]$} \\
\hline Anxiety (GAD-7) & 1.04 & [1.01-1.07] & 1.03 & [1.01-1.05] & 1.04 & [1.02-1.06] \\
\hline
\end{tabular}


Table 1 (continued)

\begin{tabular}{|c|c|c|c|c|c|c|}
\hline & \multicolumn{2}{|c|}{ Model IV: Helpline/online services } & \multicolumn{2}{|c|}{$\begin{array}{l}\text { Model V: } \\
\text { Self-care/help }\end{array}$} & \multicolumn{2}{|c|}{ Model VI: family/friend } \\
\hline & OR & $95 \% \mathrm{CI}$ & OR & $95 \% \mathrm{CI}$ & OR & $95 \% \mathrm{CI}$ \\
\hline Coping: problem-focussed & 1.18 & {$[0.92-1.51]$} & 1.80 & {$[1.53-2.11]$} & 1.12 & {$[0.96-1.30]$} \\
\hline Coping: emotion-focussed & 1.16 & {$[0.93-1.45]$} & 1.47 & {$[1.32-1.64]$} & 1.00 & {$[0.89-1.12]$} \\
\hline Coping: avoidant & 1.18 & {$[0.97-1.43]$} & 0.93 & {$[0.81-1.05]$} & 0.92 & {$[0.80-1.07]$} \\
\hline Coping: socially supported & 1.71 & {$[1.43-2.03]$} & 1.72 & {$[1.55-1.91]$} & 4.19 & {$[3.73-4.72]$} \\
\hline Personality: openness to experience & 0.98 & {$[0.80-1.19]$} & 1.77 & {$[1.57-2.01]$} & 1.13 & {$[1.00-1.29]$} \\
\hline Personality: conscientiousness & 0.95 & {$[0.81-1.11]$} & 0.90 & {$[0.81-1.00]$} & 0.93 & {$[0.84-1.04]$} \\
\hline Personality: extraversion & 1.15 & {$[0.96-1.37]$} & 0.88 & {$[0.80-0.97]$} & 1.35 & {$[1.21-1.50]$} \\
\hline Personality: agreeableness & 1.41 & {$[1.00-2.00]$} & 1.20 & {$[0.98-1.46]$} & 1.30 & {$[1.05-1.60]$} \\
\hline Personality: neuroticism & 1.27 & {$[1.04-1.56]$} & 1.08 & {$[0.97-1.20]$} & 1.22 & {$[1.09-1.36]$} \\
\hline
\end{tabular}

$p<0.05$ in bold text

engaging in self-care activities were the most commonly used approaches for people to support good mental health during the pandemic, followed by use of medication. On the contrary, formal approaches such as talking to mental health professionals, a GP or other health professional, and using helpline or online services were the least commonly used approaches. Some groups at higher risk of mental ill-health during lockdown did make higher use of support strategies, including women, people living alone, people with a preexisting mental health diagnosis or people who were experiencing higher levels of anxiety or depression. However, there were also other groups who were at risk of poorer mental health and who also were less likely to access support strategies including individuals with lower educational qualifications.

Some groups such as socially disadvantaged individuals had already been identified as being at a higher risk for experiencing difficulties in accessing mental health services before the pandemic [21]. It has been shown that people with lower education level are more likely to encounter acceptability barriers, where they prefer to manage their mental health themselves, do not know how or where to get help, and are afraid to ask for help [21]. Compounding these existing inequalities, people from lower socio-economic backgrounds experienced more adversities during the pandemic (e.g. loss of work, unable to pay bills, unable to access sufficient food ad medication) [22] and had poorer trajectories of depression and anxiety [6]. As such, it is known that this group was in more need of mental health support. So it is concerning that they had lower levels of engagement with both formal and informal mental health support. This highlights the need for more mental health interventions targeted at lower socio-economic groups with the aim of reducing the exacerbation of mental health related inequalities as the pandemic continues. On the contrary, some groups are found to have higher use of support strategies during the pandemic, including women and people living alone. This may explain why these groups, despite having higher levels of depression and anxiety when lockdown commenced, experienced a faster recovery in their mental health during the lockdown period [6].

As well as differences in the overall cumulative number of approaches used, there were also differences in the use of specific mental health approaches amongst different groups. Demographically, while older adults were more likely to take medication to support mental health, younger adults were more likely to use alternative approaches to maintain good mental health (such as talking to mental health professionals, using helpline or online services, self-care activities and talking to family/friends). This difference can be explained by cohort effects, with younger generations being more used to communicating their emotions and mental health issues with others in the wake of campaigns such as Mental Health Matters and Heads Together [23, 24]. In line with previous studies, women were more likely than men to support their mental health via informal strategies, including engaging in self-care activities and sharing their concerns and worries with family/friends [25, 26]. However, while we found that people from an ethnic minority background were less likely to manage their mental health through medication (in line with previous literature which show that the prescription of mental health medication was lower amongst minority ethnic groups [27, 28]), they were more likely to use helpline or online services. We also found that people who are socially isolated or lonely, and those with higher levels of depression or anxiety were more likely to take both formal (e.g. medication) and informal (e.g. speaking to family/friends) mental health approaches. This is in line with previous literature that mental health services are commonly used by people with a mental health diagnosis or those who are struggling with emotional problems [29, 30]. Finally, individuals with higher educational levels and income were also less likely to 
Fig. 2 Incident risk ratios and $95 \%$ confidence intervals from the Poisson regression model including all predictors $(N=26,720)$

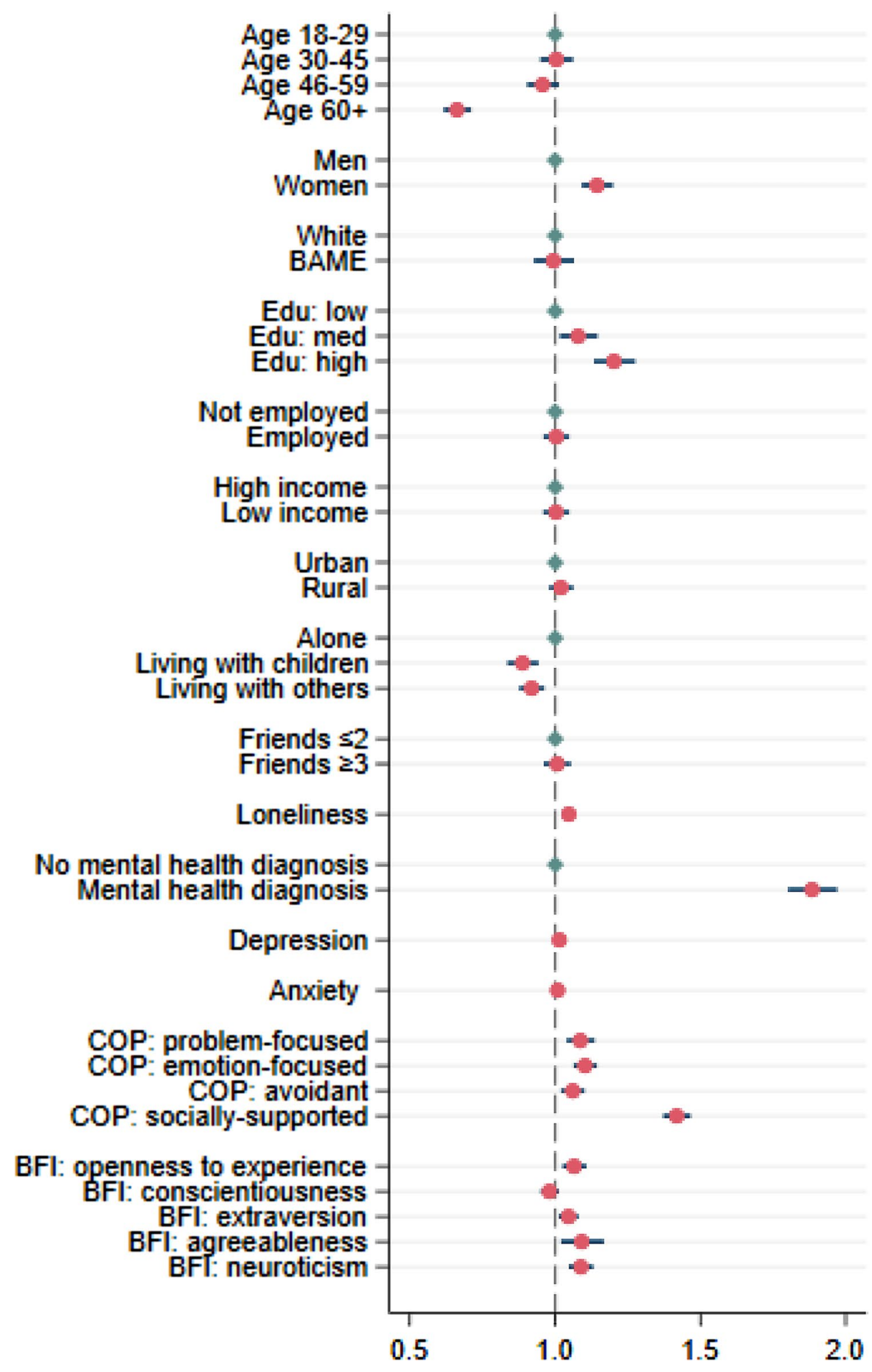

use medication to support mental health. This finding corresponds with previous studies conducted before the pandemic, which found that people without qualifications were more likely to use antidepressant drugs and anti-anxiety medication [31, 32].
Our study has also shown some further results that have received less attention in previous literature. For example, we found that mental health approaches vary depending on people's coping strategies when they experience stress, and their personality. Specifically, people who employed 
problem-focussed coping strategies were more likely to engage in self-care activities to manage their mental health during the pandemic, whereas those who employed avoidant coping strategies were more likely to use medication. This could highlight the desire for faster temporary relief from overwhelming emotions amongst people with avoidant coping styles [33], in comparison to a willingness to more proactively seek to control and manage symptoms amongst people with problem-focussed coping styles [34]. Further, we found that use of formal mental health support did not vary by personality, but use of informal support did. Notably, while sharing concerns/worries with family/friends were a common strategy for people of nearly all types of personality (except for conscientiousness), people who were more agreeable or neurotic tended to seek external help from helpline/online services or from family/friends. Alternatively, people who were more open were more likely to engage in self-care. The results are supported by a previous metaanalysis study which shows that, in particular, neuroticism was positively associated with emotional support for coping strategies, whereas high levels of openness were related to problem-solving strategies [35].

This research used a large, well-stratified sample weighted to population proportions to identify the predictors of different approaches people take to support their mental health. However, there are some limitations to the study. First, the data on use of mental health support strategies relied on participants' self-reports, so could be affected by recall bias or an unwillingness to disclose this information. However, as questions were asked weekly (limiting the length of time people had to remember their actions for) and responses were anonymous, these sources of bias are anticipated to be small. Second, our study asked participants about six different types of formal and informal mental health support strategies, but it is possible that participants might have also been using alternative approaches to manage their mental health not captured in our questions, including risky behaviours such as substance use. In addition, data on participants' previous use of mental health services prior to the pandemic are not available, so it is not known whether participants started using specific strategies during the pandemic or whether these were simply a continuation of previous habits. Further, in line with previous studies [36], we found that people from ethnic minority backgrounds were less likely to access structured mental health services. However, due to limitations in statistical power, we were only able to explore ethnicity as a binary in these analyses as there were only $4 \%$ of participants who identified themselves as ethnic minority in the raw data. We recognise that such simple categorisation likely misses nuances of experience in specific ethnic groups. On a related note, it remains unclear what the barriers to accessing services were amongst people from ethnic minority backgrounds (e.g. language barriers, discrimination or social stigma) [37-39]. Finally, our analyses did not include details of specific psychiatric diagnosis. Further work is needed to understand how service usage varied for specific clinical groups.

Given limited resources in mental health services and given the growing mental health problems during and after the COVID-19 pandemic, it is important to understand what approaches people employ to support good mental health and to identify which groups are not receiving adequate mental health support. The results of this study suggest that certain groups require more specific mental health support, in particular people with higher levels of loneliness, depression, and anxiety during the pandemic and people with a diagnosed mental health condition for which they may have been receiving support before the pandemic. It is promising that talking to family/friends about mental health and selfcare activities were the most commonly used strategies during lockdown as it suggests a recognition of the challenges to mental health posed by the pandemic and an openness in discussing mental health issues. However, it is notable that this was also the case specifically amongst people with existing diagnosed mental health conditions. Such strategies may be insufficient for the management of more severe types of mental illness and this, combined with the identification of groups facing more barriers to mental health support, suggests the importance for clinicians and policymakers of developing more specific programmes as the pandemic continues to reach those most in need of mental health support.

Supplementary Information The online version contains supplementary material available at https://doi.org/10.1007/s00127-021-02105-w.

Author contributions DF and FB conceived and designed the study. FB analysed the data and FB, HWM and DF wrote the first draft. All authors provided critical revisions. All authors read and approved the submitted manuscript.

Funding This COVID-19 Social Study was funded by the Nuffield Foundation [WEL/FR-000022583], but the views expressed are those of the authors and not necessarily the Foundation. The study was also supported by the MARCH Mental Health Network funded by the Cross-Disciplinary Mental Health Network Plus initiative supported by UK Research and Innovation [ES/S002588/1]. DF was funded by the Wellcome Trust [205407/Z/16/Z]. The researchers are grateful for the support of a number of organisations with their recruitment efforts including: the UKRI Mental Health Networks, Find Out Now, UCL BioResource, HealthWise Wales, SEO Works, FieldworkHub, and Optimal Workshop. The funders had no final role in the study design; in the collection, analysis and interpretation of data; in the writing of the report; or in the decision to submit the paper for publication. All researchers listed as authors are independent from the funders and all final decisions about the research were taken by the investigators and were unrestricted. All authors had full access to all of the data (including statistical reports and tables) in the study and can take responsibility for the integrity of the data and the accuracy of the data analysis. 
Data availability Anonymous data will be made available following the end of the pandemic.

Code availability Stata syntax are available from the authors upon request.

\section{Declarations}

Conflict of interest All authors declare no conflicts of interest.

Ethical approval Ethical approval for the COVID-19 Social Study was granted by the UCL Ethics Committee.

Informed consent All participants provided fully informed consent. The study is GDPR compliant.

Open Access This article is licensed under a Creative Commons Attribution 4.0 International License, which permits use, sharing, adaptation, distribution and reproduction in any medium or format, as long as you give appropriate credit to the original author(s) and the source, provide a link to the Creative Commons licence, and indicate if changes were made. The images or other third party material in this article are included in the article's Creative Commons licence, unless indicated otherwise in a credit line to the material. If material is not included in the article's Creative Commons licence and your intended use is not permitted by statutory regulation or exceeds the permitted use, you will need to obtain permission directly from the copyright holder. To view a copy of this licence, visit http://creativecommons.org/licenses/by/4.0/.

\section{References}

1. Potter C, Parrish S, Eli K, Lavis A, Nowicka P, Ulijaszek S (2020) Changes in mental health, eating and physical activity in England across Covid-19 pandemic lockdown. https://438d19e0-717949d4-9417-1beadf30680c.filesusr.com/ugd/957749_ce31fa76a6 c247b6a28924a41f2431ef.pdf?index =true

2. Pierce M, Hope H, Ford T, Hatch S, Hotopf M, John A et al (2020) Mental health before and during the COVID-19 pandemic: a longitudinal probability sample survey of the UK population. Lancet Psychiatry 1-10. www.thelancet.com/psychiatryPublishedonline

3. Xiang YT, Yang Y, Li W, Zhang L, Zhang Q, Cheung T et al (2020) Timely mental health care for the 2019 novel coronavirus outbreak is urgently needed. Lancet Psychiatry. 7(3):228-229. https://www.ncbi.nlm.nih.gov/pmc/articles/PMC7128153/

4. Brooks SK, Webster RK, Smith LE, Woodland L, Wessely S, Greenberg N et al (2020) The psychological impact of quarantine and how to reduce it: rapid review of the evidence. Lancet 395(10227):912-920. https://doi.org/10.1016/S0140-6736(20) 30460-8

5. Iob E, Frank P, Steptoe A, Fancourt D (2020) Levels of severity of depressive symptoms among at-risk groups in the UK during the COVID-19 pandemic. JAMA Netw Open 3(10):e2026064. https://jamanetwork.com/journals/jamanetworkopen/fullarticle/ 2772163

6. Fancourt D, Steptoe A, Bu F (2020) Trajectories of depression and anxiety during enforced isolation due to COVID-19: longitudinal analyses of 59,318 adults in the UK with and without diagnosed mental illness. medRxiv. https://doi.org/10.1101/2020.06.03. 20120923
7. Forbes MK, Krueger RF (2019) The great recession and mental health in the United States. Clin Psychol Sci 7(5):900-913. https:// doi.org/10.1177/2167702619859337

8. Holmes EA, O'Connor RC, Perry VH, Tracey I, Wessely S, Arseneault L et al (2020) Multidisciplinary research priorities for the COVID-19 pandemic: a call for action for mental health science. Lancet Psychiatry 7(6):547-560. www.thelancet.com/psychiatry

9. National Health Service (NHS) (2020) Mental wellbeing while staying at home I every mind matters I one you. https://www. nhs.uk/oneyou/every-mind-matters/coronavirus-covid-19-stayi ng-at-home-tips/

10. Samaritans (2020) How has coronavirus affected our callers? https://www.samaritans.org/about-samaritans/research-policy/ understanding-our-callers-during-covid-19-pandemic/how-hascoronavirus-affected-our-callers/

11. Packness A, Halling A, Simonsen E, Waldorff FB, Hastrup LH (2019) Are perceived barriers to accessing mental healthcare associated with socioeconomic position among individuals with symptoms of depression? Questionnaire-results from the Lolland-Falster Health Study, a rural Danish population study. BMJ Open 9:23844. http://bmjopen.bmj.com/

12. Sentell T, Shumway M, Snowden L (2007) Access to mental health treatment by English language proficiency and race/ethnicity. J Gen Intern Med 22:289-293. https://doi.org/10.1007/ s11606-007-0345-7

13. Iob E, Steptoe A, Fancourt D (2020) Abuse, self-harm and suicidal ideation in the UK during the COVID-19 pandemic. Br J Psychiatry 217:543-546. https://doi.org/10.1192/bjp.2020.130

14. Russell D, Peplau LA, Cutrona CE (1980) The revised UCLA Loneliness Scale: concurrent and discriminant validity evidence. J Pers Soc Psychol 39(3):472-480. https://pubmed.ncbi. nlm.nih.gov/7431205/

15. Kroenke K, Spitzer RL (2002) The PHQ-9: a new depression diagnostic and severity measure. Psychiatr Ann 32(9):1-7. http://jacobimed.org/public/Ambulatory_files/mlove/Curri culumWomenandGeri/Depression/Depression articles/PHQ9ReviewKroenke.pdf

16. Spitzer RL, Kroenke K, Williams JBW, Löwe B (2006) A brief measure for assessing generalized anxiety disorder: the GAD-7. Arch Intern Med 166(10):1092-1097

17. Soto CJ, John OP (2017) The next Big Five Inventory (BFI-2): developing and assessing a hierarchical model with 15 facets to enhance bandwidth, fidelity, and predictive power. J Pers Soc Psychol 113(1):117-143. https://pubmed.ncbi.nlm.nih.gov/ 27055049/

18. Carver CS (1997) You want to measure coping but your protocol's too long: consider the brief COPE. Int J Behav Med 4(1):92-100. https://doi.org/10.1207/s15327558ijbm0401_6

19. Nahlen Bose C, Bjorling G, Elfstrom ML, Persson H, Saboonchi $F(2015)$ Assessment of coping strategies and their associations with health related quality of life in patients with chronic heart failure: the brief COPE restructured. Cardiol Res 6(2):239-248. https://doi.org/10.14740/cr385w

20. Office for National Statistics (ONS) (2020) Population estimates for the UK, England and Wales, Scotland and Northern Ireland. Office for National Statistics. https://www.ons.gov.uk/peoplepopu lationandcommunity/populationandmigration/populationestim ates/bulletins/annualmidyearpopulationestimates/mid2018

21. Steele L, Dewa C, Lee K (2007) Socioeconomic status and selfreported barriers to mental health service use. Can J Psychiatry 52(3):201-206

22. Wright L, Steptoe A, Fancourt D (2020) Are we all in this together? Longitudinal assessment of cumulative adversities by socioeconomic position in the first 3 weeks of lockdown in the UK. J Epidemiol Community Health 74(9):683-688. https://doi. org/10.1136/jech-2020-214475 
23. Mental Health Matters (2020) Mental Health Matters. https:// www.mhm.org.uk/

24. Heads Together (2020) Heads Together. https://www.headstoget her.org.uk/

25. Eschenbeck H, Kohlmann CW, Lohaus A (2007) Gender differences in coping strategies in children and adolescent. Artic J Individ Differ 28(1):18-26. https://www.researchgate.net/publication/ 240218695

26. Ptacek JT, Smith RE, Dodge KL (1994) Gender differences in coping with stress: when stressor and appraisals do not differ. Personal Soc Psychol Bull 20(4):421-430. https://doi.org/10.1177/ 0146167294204009

27. Sreeharan V, Madden H, Lee JT, Millett C, Majeed A (2013) Improving access to psychological therapies and antidepressant prescribing rates in England: a longitudinal time-series analysis. Br J Gen Pract. e649-e653. https://bjgp.org/content/bjgp/63/614/ e649.full.pdf

28. Walters P, Ashworth M, Tylee A (2008) Ethnic density, physical illness, social deprivation and antidepressant prescribing in primary care: ecological study. Br J Psychiatry. 193:235-239. www. ic.nhs.uk/services/qof

29. Morgan K, Chakkalackal L, Cyhlarova E (2012) Life Lines: evaluation of mental health helplines. Mental Health Foundation. https://www.mentalhealth.org.uk/sites/default/files/life_lines.pdf

30. Garland AF, Lau AS, Yeh M, McCabe KM, Hough RL, Landsverk JA (2005) Racial and ethnic differences in utilization of mental health services among high-risk youths. Am J Psychiatry. 162(7):1336-1343. http://ajp.psychiatryonline.org

31. Boyle S, Murphy J, Rosato M, Boduszek D, Shevlin M (2020) Predictors of antidepressant use in the English population: analysis of the Adult Psychiatric Morbidity Survey. Irish J Psychol Med 37(15):23
32. Schofield MJ, Khan A (2014) Predictors of prescribed medication use for depression, anxiety, stress, and sleep problems in midaged Australian women. Soc Psychiatry Psychiatr Epidemiol 49:1835-1847

33. Herman-Stahl MA, Stemmler M, Petersen AC (1995) Approach and avoidant coping: implications for adolescent mental health. J Youth Adolesc 26(6):649-665

34. Lazarus RS, Folkman S (1991) The concept of coping. In: Monat A, Lazarus RS (eds) Stress and coping: an anthology. Columbia University Press, New York, pp 189-206. https://psycnet.apa.org/ record/1991-97822-009

35. Connor-Smith JK, Flachsbart C (2007) Relations between personality and coping: a meta-analysis. J Pers Soc Psychol 9(6):1080 1107. https://doi.org/10.1037/0022-3514.93.6.1080.supp

36. BAME and mental health (2020). Mental Health Foundation. https://www.mentalhealth.org.uk/a-to-z/b/black-asian-and-minor ity-ethnic-bame-communities

37. Chandra A, Minkovitz CS (2006) Stigma starts early: gender differences in teen willingness to use mental health services. J Adolesc Heal 38(6):754.e1-754.e8

38. Memon A, Taylor K, Mohebati LM, Sundin J, Cooper M, Scanlon $\mathrm{T}$ et al (2016) Perceived barriers to accessing mental health services among black and minority ethnic (BME) communities: a qualitative study in Southeast England. BMJ Open 6(11):e012337. http://bmjopen.bmj.com/

39. Ae SKT, Wang J (2008) Ethnic differences in mental health service use among White, Chinese, South Asian and South East Asian populations living in Canada. Soc Psychiatry Psychiatr Epidemiol 43:866-871 